\title{
Thirteen-year follow-up of parasellar intravascular papillary endothelial hyperplasia successfully treated by surgical excision: case report
}

\author{
Sook Young Sim, MD, PhD, ${ }^{1}$ Yong Cheol Lim, MD, ${ }^{2}$ Keun Soo Won, MD, PhD, ${ }^{3}$ \\ and Kyung Gi Cho, MD, PhD
}

\begin{abstract}
1Department of Neurosurgery, Inje University Seoul Paik Hospital, Seoul; ${ }^{2}$ Department of Neurosurgery, Ajou University School of Medicine, Suwon; and ${ }^{3}$ Department of Neurosurgery, Bundang CHA Medical Center, CHA University, College of Medicine, Seongnam, Korea
\end{abstract}

\begin{abstract}
Intracranial intravascular papillary endothelial hyperplasia (IPEH) is very rare, and to our knowledge long-term follow-up results have not been previously published. An 11-year-old boy presented with a 6 -month history of progressive visual impairment in the right eye. Magnetic resonance imaging revealed a well-enhanced, large parasellar mass involving the cavernous sinus, right frontal skull base, and ethmoid and sphenoid sinuses. Frontotemporal craniotomy and subtotal resection were performed, and the diagnosis of IPEH was confirmed. The mass increased in size during the following 3 months. A second operation was performed via frontotemporal craniotomy combined with a transsphenoidal approach, and gross-total resection of the tumor was achieved. Adjuvant radiotherapy (5040 cGy) and chemotherapy with interferon were administered. The patient's visual symptoms improved, and there was no recurrence during a 13-year follow-up period. The results of this case indicate that intracranial IPEH can recur with subtotal resection; however, optimal resection with multimodal adjuvant treatment can control the disease for many years, if not permanently.
\end{abstract}

http://thejns.org/doi/abs/10.3171/2014.9.PEDS13518

KEY WORDS hemangioendothelioma; intravascular papillary endothelial hyperplasia; intracranial tumor; vascular tumor; oncology

I NTRAVASCULAR papillary endothelial hyperplasia (IPEH) is an excessive proliferation of endothelial cells within the space of a preexisting vessel or vascular malformation or within an organizing hematoma. ${ }^{3,6,11,14,19}$ IPEH can occur in various parts of the body, including a number of internal organs (gastrointestinal tract, kidney, liver, lung, uterus, etc.), head and neck structures (nasal cavity, pharynx, and larynx), and most commonly, in skin and subcutaneous tissue of the upper extremities. . $^{3,6,14,19}$ While extracranial IPEH usually presents as a slow-growing painful benign nodule, ${ }^{6,14}$ IPEH involving intracranial structures sometimes leads to serious local compressive symptoms, such as cranial nerve palsy, or a potentially fatal increase in intracranial pressure. To date, only 22 cases of intracranial IPEH have been reported. ${ }^{1-9,12-19}$ Because of its rarity, the natural history and treatment guidelines for intracranial IPEH have not been fully elucidated. Moreover, to our knowledge long-term follow-up data after surgical treatment of intracranial IPEH have not previously been pub- lished. In this report, we present a case of parasellar IPEH that has been successfully treated with complete resection and multimodal adjuvant treatment and has been followed up for 13 years.

\section{Case Report}

History and Examination

An 11-year-old boy presented with a 6-month history of progressive visual impairment in his right eye. He could recognize hand movement at a distance of no further than $15 \mathrm{~cm}$ with that eye, and ophthalmological examination revealed significant papilledema. All other cranial nerve functions were within normal limits. Noncontrast brain CT revealed a well-demarcated high-density mass in the ethmoid and sphenoid sinuses, extending into the right cavernous sinus and suprasellar area, with bony erosions in each sinus. Magnetic resonance imaging revealed a wellenhanced, lobulated mass in the ethmoid and sphenoid 
sinuses, involving the sella turcica and right cavernous sinus and extending into the right frontal skull base and right frontal lobe. The mass was of mixed-to-high signal intensity on T2-weighted MR images, and of isointense signal intensity on T1-weighted images. The right cavernous segment of the internal carotid artery was encased in the tumor. The right optic nerve was also encroached upon by the intracranial mass and was significantly displaced upward. There were several circular areas of signal hypointensity inside the tumor. The mass was approximately $5 \times 3 \times 3 \mathrm{~cm}$ in size, well demarcated from the surrounding tissue, and had no peritumoral edema, which strongly suggested that the lesion was a benign extraaxial tumor (Fig. 1). Cerebral angiography revealed a massive tumor blush from the internal and external carotid arteries.

\section{First Operation}

Staged operations were planned such that a transcranial approach would be followed by a transsphenoidal approach. Right frontotemporal craniotomy with an extradural approach was initially performed to debulk the mass. The anterior clinoid process was completely removed using a high-speed air drill, and the temporal dura mater was removed to expose the outer wall of the cavernous sinus and the tumor mass. The mass was darkish red and consisted of both fresh and old organizing thrombi. The tumor was highly vascular and caused massive intraoperative bleeding; in addition, it adhered tightly to the ipsilateral internal carotid artery and optic nerve. A meticulous and diligent procedure resulted in total removal of the tumor around the sella turcica and within the cavernous sinus. Most of the tumor mass which extended down into the ethmoid and sphenoid sinuses was removed; however, $10 \%$ of the tumor mass in this region could not be resected because of poor surgical visualization.

\section{Pathological Findings}

On histopathological examination, the tissue mass was found to contain numerous papillae with hyaline cores surrounded by monolayered endothelial hyperplasia (Fig. $2 \mathrm{~A}$ and $\mathrm{B}$ ). The endothelium had no malignant features such as anaplasia or necrosis. Intravascular hemorrhage and organized thrombi were also observed. Immunohistochemical staining with CD34 showed positive reactivity, reflecting a complex vascular network of the tissue mass (Fig. 2C). All of these findings indicated the diagnosis of IPEH. The Ki-67 labeling index was over $30 \%$.

\section{Postoperative Course}

Immediately after the operation, the vision in the patient's right eye improved slightly, and he was able to recognize a finger count at a distance of $15 \mathrm{~cm}$. However, a 3-month follow-up MRI study, which was intended to aid in planning the second stage operation, revealed that the residual mass had grown dramatically to its previous size (Fig. 3).

\section{Second Operation and Postoperative Course}

A second operation was carried out using a combined transcranial and transsphenoidal approach. The mass was totally removed (Fig. 4A-C), and the surgical damage to
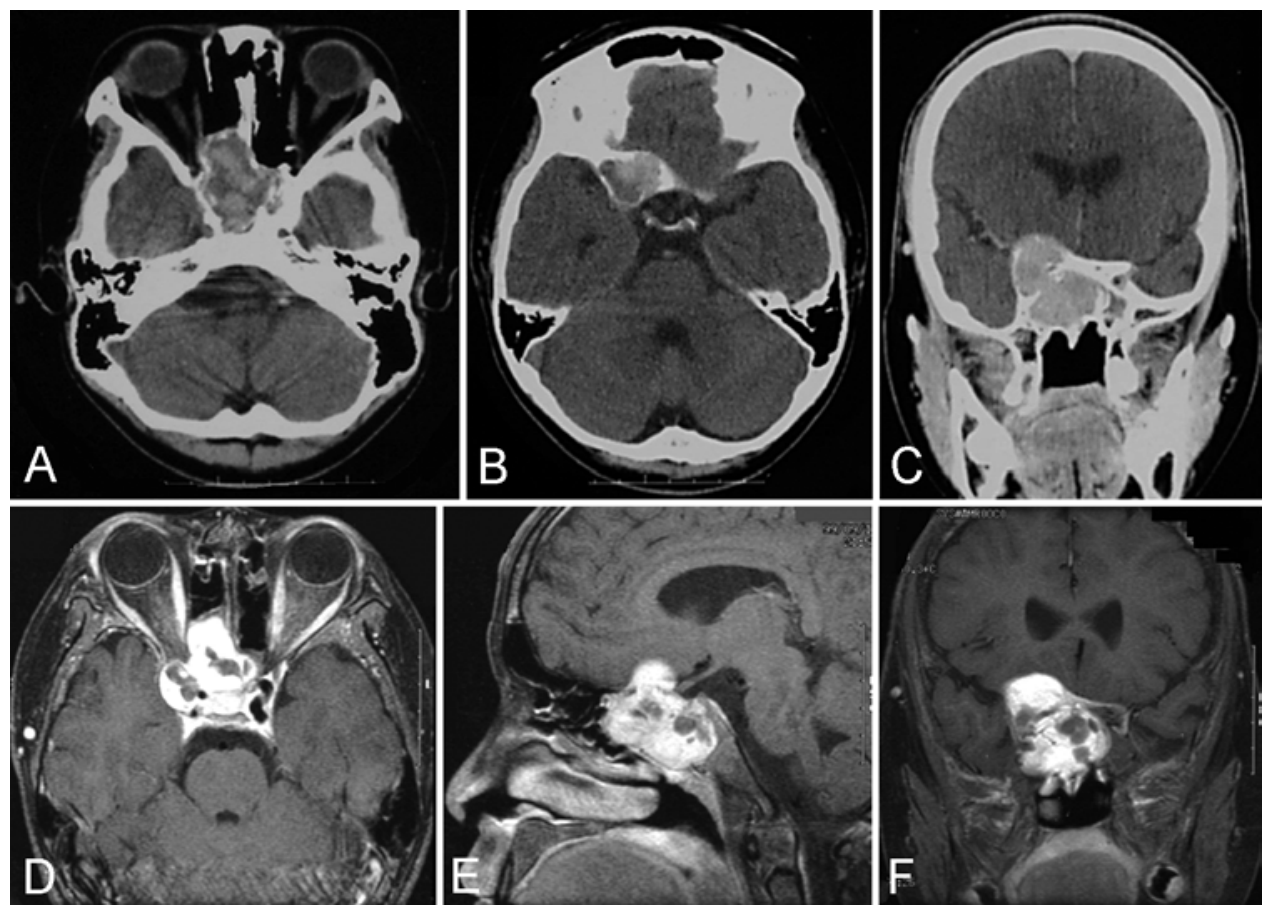

FIG. 1. A-C: Noncontrast axial (A and B) and coronal (C) CT images showing a well-demarcated high-density mass in the ethmoid and sphenoid sinuses extending into the right cavernous sinus and suprasellar area. Note that the ethmoid and sphenoid sinuses were somewhat destroyed by the mass (C). D-F: Gd-enhanced T1-weighted axial (D), sagittal (E), and coronal (F) MR images revealing a well-enhanced, lobulated mass in the ethmoid and sphenoid sinuses involving the sella turcica and the right cavernous sinus that extended into the right frontal base. The right internal carotid artery and optic nerve were affected by the mass (D). 
the anterior skull base was reconstructed using the rectus abdominis muscle flap in which the superficial temporal artery and vein were anastomosed. The patient recovered well from surgery, with a transient limitation of lateral gaze in his right eye. His best-corrected visual acuity in the right eye improved substantially, which enabled him to count fingers at a $30-\mathrm{cm}$ distance. Postoperatively, a total dose of 5040 cGy of external radiation therapy was administered, followed by 6 cycles of interferon therapy. The patient was followed up with serial MRI, and as of the 13year follow-up examination, no regrowth of the tumor has been seen (Fig. 4D-F).

\section{Discussion}

IPEH was first described by Pierre Masson, in 1923, as an intravascular papillary proliferation of endothelial cells in a lumen of an inflamed hemorrhoidal plexus; he named it "vegetant intravascular hemangioendothelioma." Since then, this rare disease entity has been described under many different names, such as Masson tumor, IPEH, Masson vegetant intravascular hemangioendothelioma, Masson pseudoangiosarcoma, intravascular angiomatosis, and Masson's hemangioma. ${ }^{1-4,12,14,16-19}$ Among these many names for this condition, IPEH is increasingly used, as it directly describes the disease pathology., , $^{3-10,13,15}$

IPEH was originally considered as a neoplasm, but it is currently regarded as an unusual nonneoplastic, reactive endothelial proliferation associated with organizing thrombi. These masses can occur either within the normal intravascular space (primary or pure type), within a preexisting vascular malformation (secondary or mixed type), or less commonly, in extravascular hematomas (undetermined or extravascular type). . $^{3,6,9,14,19}$

Reported cases, including the present one, indicate that the age at presentation of symptoms of intracranial IPEH can range from 2 days to 75 years of age, with biphasic peaks in the 2nd to 3rd decade and in the 6th decade of life (Table 1). There is a strong female predominance (19 [82.6\%] of 23 reported cases) in the incidence of intracranial IPEH, and a less obvious female predominance in IPEH at other locations. ${ }^{6}$

Intracranial IPEH can occur at the base of the skull, in the brain parenchyma, and in the intravascular spaces within the skull. Intracranial IPEHs most commonly occur in the skull base $(\mathrm{n}=13[56.5 \%])^{2,3,8,13-16,19}$ and frequently involve parasellar areas $(\mathrm{n}=9)^{3,8,14,15}$ and petrous bone (n $=4) .{ }^{1,2,16,19}$ IPEH occurring in parasellar areas frequently include the cavernous sinus, sometimes extending into the superior orbital fissure and orbit. ${ }^{13-15}$ IPEH in the petrous bone include lesions in the internal auditory meatus, ${ }^{2}$ jugular foramen, ${ }^{19}$ and Meckel's cave. ${ }^{16}$ IPEH in the brain parenchyma $(n=9$ [39.1\%]) has been reported with varying prevalence in the parietal $(\mathrm{n}=4[44.4 \%]),{ }^{3-5,12}$ frontal $(\mathrm{n}=$ $3[33.3 \%]),{ }^{4,5,17}$ temporal $(\mathrm{n}=2[22.2 \%]),{ }^{12,15}$ and occipital $(\mathrm{n}=2[22.2 \%])^{5,7}$ lobes and in the posterior fossa $(\mathrm{n}=2$ [22.2\%]), cerebellum, and brainstem, ${ }^{6,14}$ including cases involving multiple lesions. Two cases $(8.7 \%)$ were confined purely to the intravascular space (in an aneurysm of the posterior inferior cerebellar artery and in the torcular herophili),, 18 without extending into the brain parenchyma

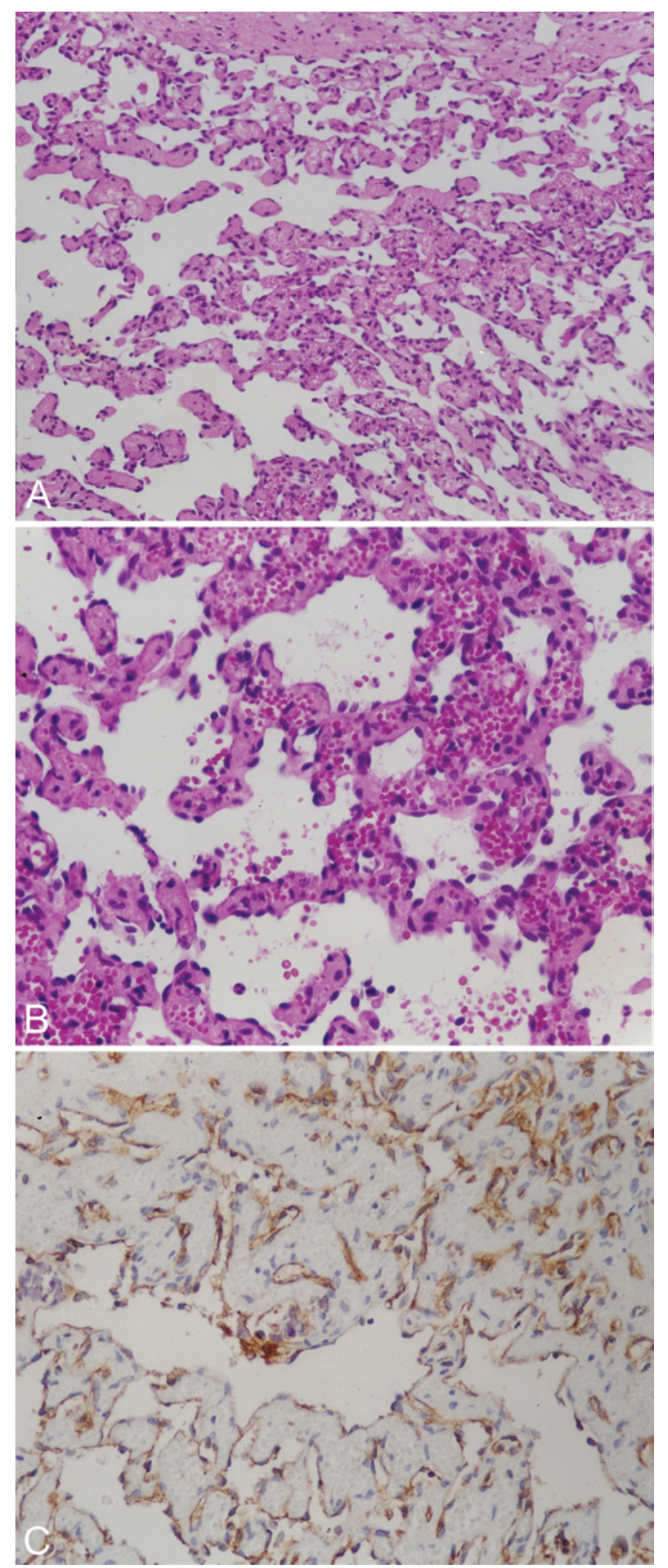

FIG. 2. A and B: Photomicrographs of $H \&$ E-stained sections. Low magnification (A) shows numerous papillary projections with hyaline cores, lined by single-layered endothelial cells. High magnification (B) shows plump endothelial cells lacking malignant features such as anaplasia or necrosis. Original magnification $\times 100(\mathrm{~A})$ and $\times 400(\mathrm{~B})$. C: Photomicrograph of immunohistochemical staining with CD34 showing positive reactivity representing a complex vascular network. Original magnfication $\times 200$. Figure is available in color online only. 

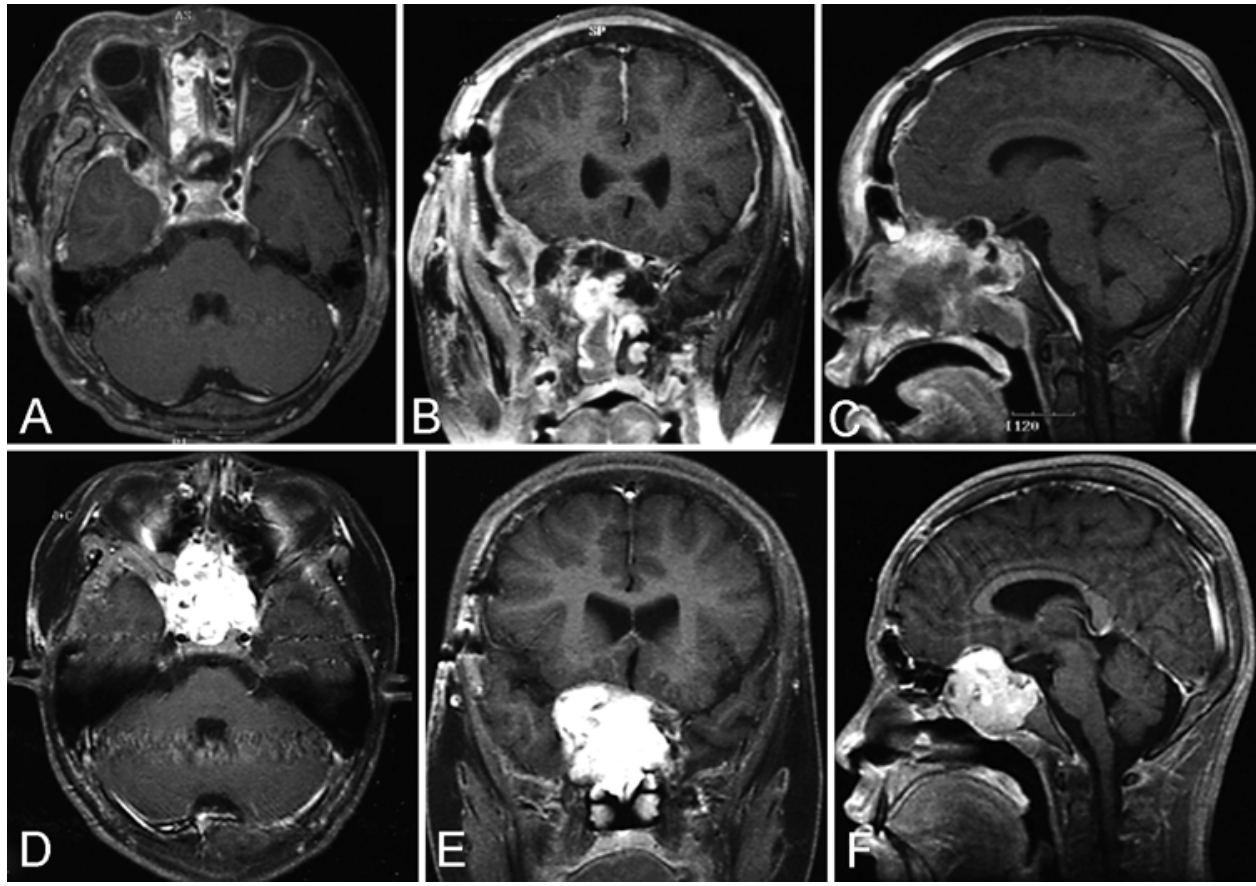

FIG. 3. A-C: Postoperative Gd-enhanced T1-weighted axial (A), coronal (B), and sagittal (C) MR images obtained after the first operation revealing successful decompression of the tumor with approximately $90 \%$ reduction of its original size. Residual tumor in the sphenoid and ethmoid sinus could not be resected completely owing to a poor surgical corridor. D-F: Follow-up Gd-enhanced T1-weighted axial (D), coronal (E), and sagittal (F) MR images obtained 3 months after the first operation revealing an increase in the residual mass.

and bony structures. Most cases were of the primary type, although in 5 cases $(21.7 \%)$, the lesions arose from preexisting vascular malformations, including cavernous malformations $(\mathrm{n}=3),{ }^{3,7,8}$ an arteriovenous malformation $(\mathrm{n}=$
1),${ }^{17}$ and a venous angioma $(\mathrm{n}=1) \cdot{ }^{13}$ Multiple intracranial IPEHs have been reported in 3 cases, ${ }^{5,12,14}$ and all were deemed primary type IPEH.

Symptoms in patients with intracranial IPEH depend
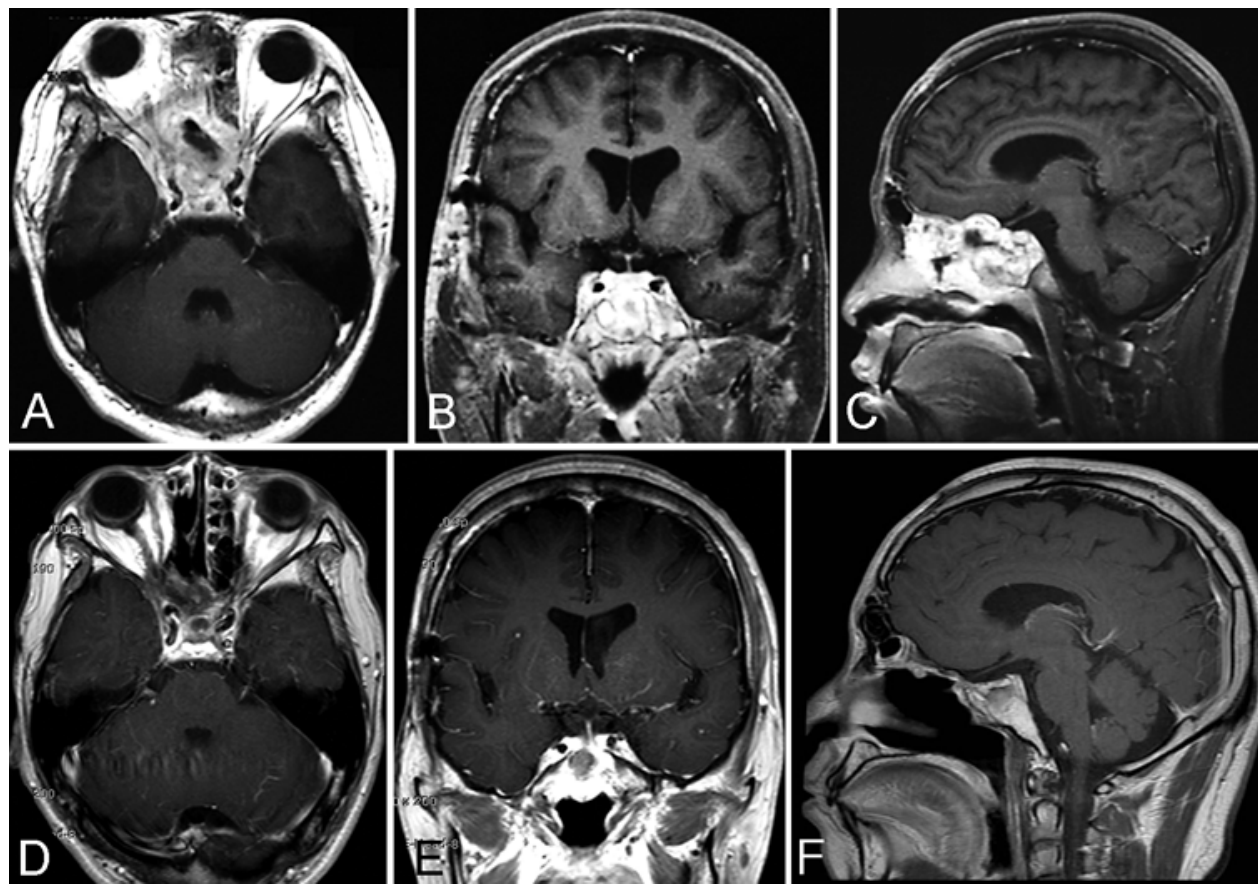

FIG. 4. A-C: Postoperative Gd-enhanced T1-weighted axial (A), coronal (B), and sagittal (C) MR images obtained after the second operation showing gross-total resection of the intracranial IPEH via a combined transcranial and transsphenoidal approach. D-F: Follow-up Gd-enhanced T1-weighted axial (D), coronal (E), and sagittal (F) MR images obtained 13-years after the second operation showing no evidence of recurrence. 


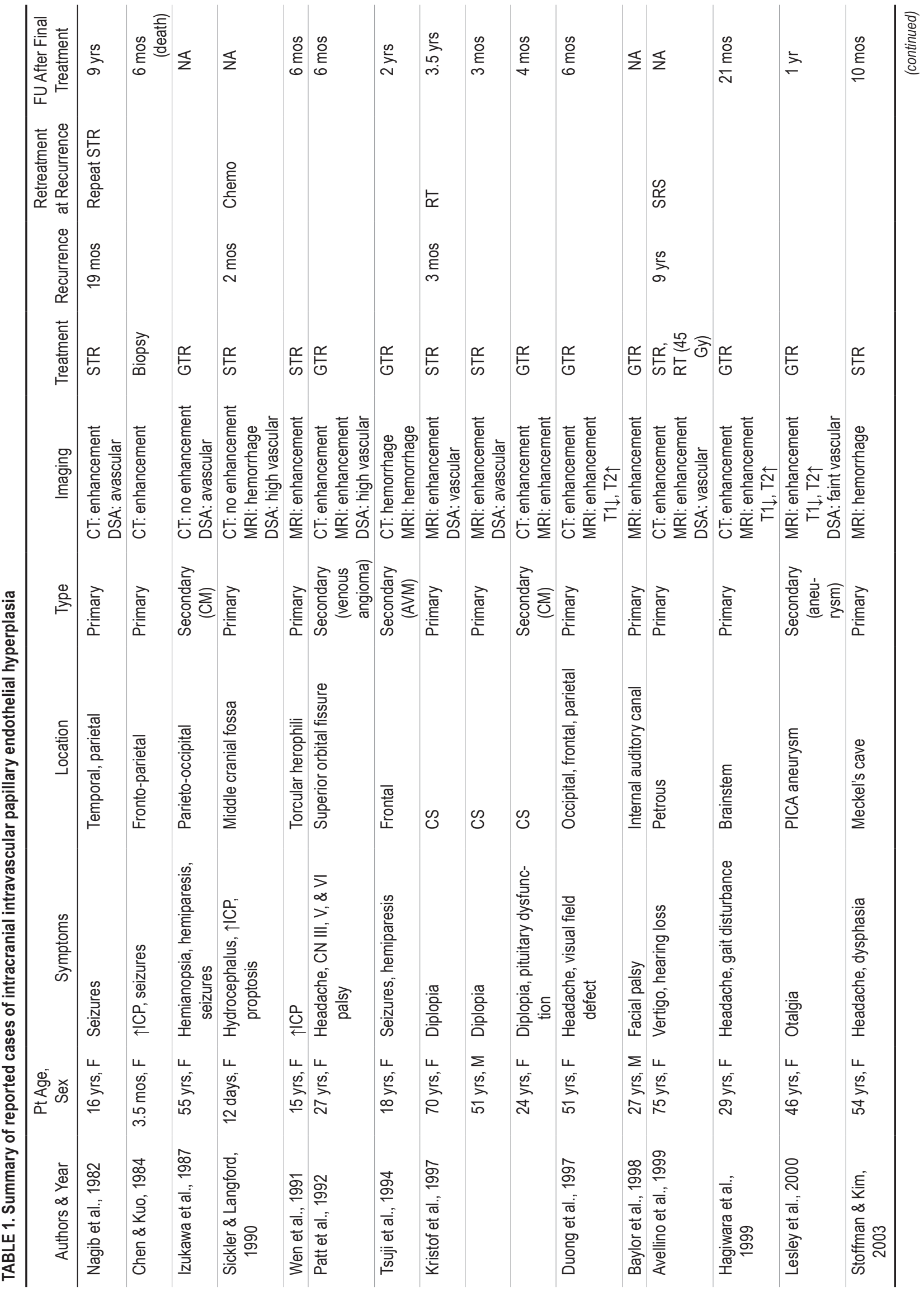




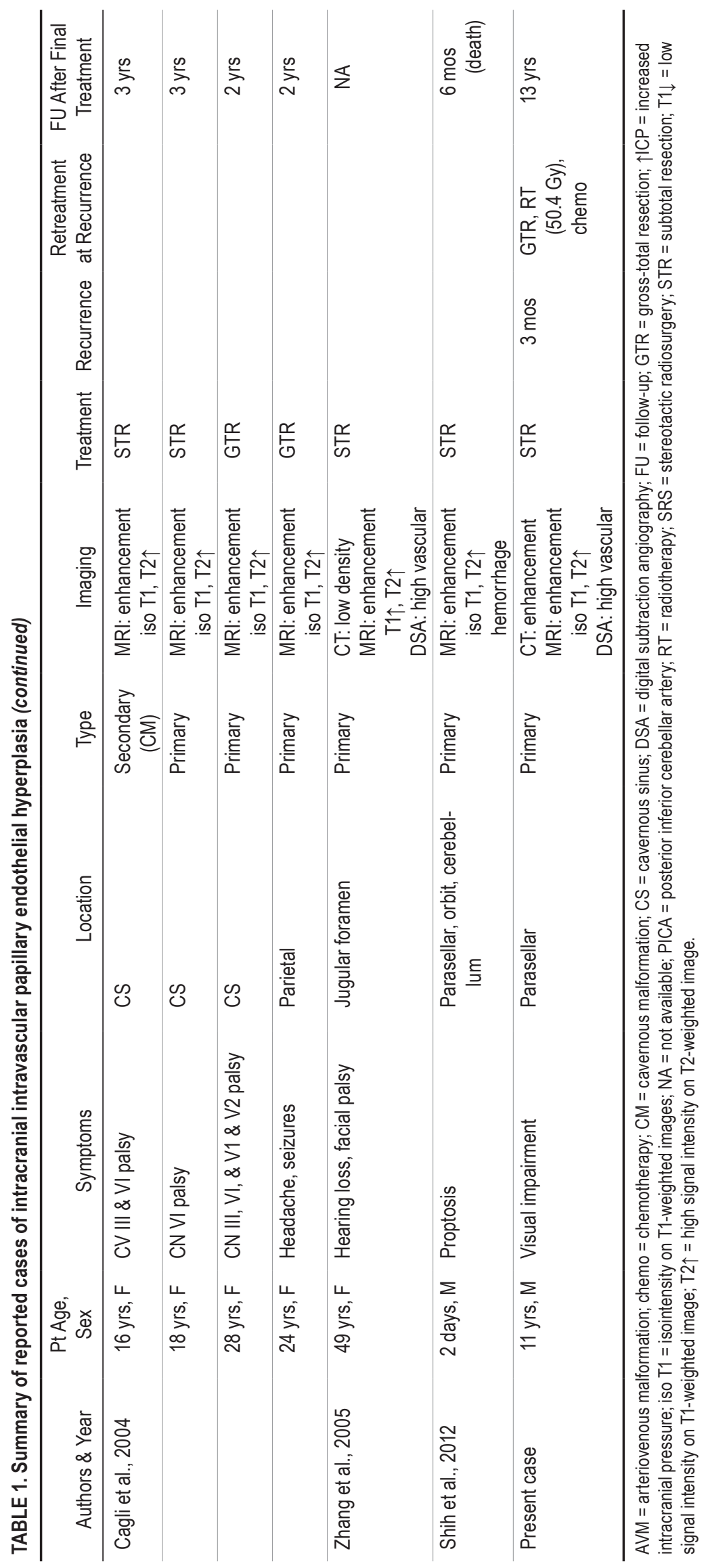


on the lesion location. Given that the most common location is the base of the skull, from which cranial nerves exit through foramina, the most common symptoms of intracranial IPEH are single or multiple cranial nerve palsies $(\mathrm{n}=12[52.2 \%]){ }^{1-3,5,7,8,13,19}$ Increased intracranial pressure is the second most common presenting manifestation $(\mathrm{n}=8[34.8 \%]) ;^{3-6,13,15,16,18}$ followed by seizure $(\mathrm{n}=$ $5[21.7 \%])^{3}, 3,7,12,17$ local mass effect, exclusive of cranial nerve effects, including proptosis, ataxia, and hemiparesis $(\mathrm{n}=5[21.7 \%]))^{6,7,14,15,17}$ and intracranial hemorrhage $(\mathrm{n}=2$ $[8.7 \%])^{7,17}$ Interestingly, intracranial hypertension secondary to venous outflow obstruction because of IPEH in the venous sinus was reported in 1 case. ${ }^{18}$

The cause and pathogenesis of IPEH continues to be debated. It is evident that IPEH is often associated with thrombosis, either in a normal vessel or in a preexisting vascular lesion. ${ }^{14}$ However, it is not clear whether endothelial proliferation precedes secondary thrombosis or, alternatively, reorganizing thrombi provoke an excessive proliferative reaction of the endothelial cells. ${ }^{3}$ One hypothesis concerning the development of IPEH is that thrombus formation and its subsequent organization result from restriction in local flow and can cause reactive endothelial proliferations with a papillary pattern. ${ }^{10}$ It is believed that organizing fibrin thrombi become transformed into papillae with hyaline cores. ${ }^{6,19}$

Although the specific cause of IPEH has not yet been elucidated, several conditions have been suggested regarding the genesis of IPEH. It may occur as a part of a syndrome such as Kasabach-Merritt syndrome in cases of infantile IPEH ${ }^{4,15}$ or systemic cavernous angiomatosis syndrome in cases of multiple IPEH. ${ }^{5,12}$ Moreover, microsurgery and radiosurgery for glioma is associated with some cases of IPEH, ${ }^{6}$ and congenital lesions are suspected in cases of neonatal IPEH. ${ }^{14}$ In addition, reproductive hormones may contribute to the larger number of cases of intracranial IPEH involving female patients. ${ }^{6}$

As in the present case, MRI findings in most cases of intracranial IPEH have revealed hyperintensities on T2weighted images, and intensities ranging from low to isointense on T1-weighted images with marked enhancement after intravenous administration of gadolinium., ${ }^{3,6,14}$ The lesions tend to have a well-demarcated margins with or without peritumoral edema. If intratumoral hemorrhage is present, it may exhibit heterogeneous signal intensity. ${ }^{14,19}$ In contrast to MRI results, findings with respect to CT density and angiographic vascular blush are more variable. ${ }^{3}$ IPEH is often accompanied by bony erosion on CT, as shown in our case (Fig. 1).

It is important to differentiate intracranial IPEH from angiomatous meningioma and angiosarcoma. Angiomatous meningioma differs from IPEH in that it contains a whirling pattern of reactive fibroblasts, clusters of arachnoid cap cells, and collagen. ${ }^{19}$ Angiosarcoma has features of malignancy such as multiple layers of endothelial cells, nuclear pleomorphisms, hyperchromatic atypical cells with mitotic figures, and necrosis. Moreover, it is almost never purely intravascular, and it infiltrates into adjacent structures. ${ }^{3,6,14,15,19}$ Immunohistochemistry for CD34 and CD31 markers and factor XIIIa has revealed patterns corresponding to the endothelial proliferation, but it has not played a major role in the differential diagnosis of other vascular tumors. ${ }^{3,14,19}$

The natural history of intracranial IPEH has not been clearly demonstrated because the condition is rare. Although IPEH has benign histological features, intracranial IPEH is clinically significant and can lead to a serious or fatal outcome because of increased intracranial pressure and local invasiveness destroying bony structures of the cranial base, which frequently affects the cranial nerves and induces intracranial hemorrhage. In particular, the prognosis is typically very poor for IPEH in infants, ${ }^{4,14,15}$ even though in one case a lesion spontaneously regressed over time. ${ }^{14}$

In addition, IPEH frequently recurs after subtotal resection. ${ }^{1,8,12,15}$ After thorough review of the previous literature, we found 5 cases of intracranial IPEH (including this case) that recurred, all of which were treated with subtotal resection or biopsy, with a mean recurrence time of 27 months after surgery. It is important to recognize that recurrence of intracranial IPEH can occur during the early postoperative period, within 2 months, ${ }^{15}$ or even up to 9 years after the initial treatment. ${ }^{1}$ Considering that all previous case reports regarding intracranial IPEH reported this rare and interesting disease at the relatively short mean follow-up period of 22.5 months (excluding the current case, in which there was 13 years of follow-up), the natural behavior of intracranial IPEH is not yet fully understood. The 17 previously reported cases of intracranial IPEH without recurrence have only been reviewed at a mean follow-up time of 12.8 months; ${ }^{2-7,9,13,14,16-19}$ therefore, the true recurrence rate for IPEH may be much higher than what is currently reported and referred to in the literature. It is of interest that this benign nonneoplastic proliferation occurs very rapidly and has a tendency to recur. There may be some differential and yet unknown aspects of disease prognosis; some patients exhibit a very aggressive course, ${ }^{8,12,15}$ whereas others do not. ${ }^{1-7,9,13,14,16-19}$ In the present case, which followed an aggressive course, the Ki-67 labeling index was much higher than expected, even compared with the index in historical control of malignant glioma. Although IPEH is a benign tumor, the findings of this case, such as high Ki-67 index and aggressive clinical behavior of rapid regrowth after subtotal resection (reaching its original size again in only 3 months), led us to apply the therapeutic strategies for malignancies such as angiosarcoma-including surgery, radiotherapy, and interferon alpha-to prevent recurrence even though we achieved gross-total resection of the tumor. We suggest that IPEHs with a high proliferating index should be treated more aggressively because of a high risk of regrowth and recurrence.

Even if it is possible to cure IPEH with complete resection, there are several factors that hinder curative resection of intracranial IPEH. These include: 1) its predilection for being located in the base of the skull and frequently involving multiple cranial nerves, 2) an association with preexisting vascular malformations or vascular structures, and 3) the possibility of being rich in vascular supply, which may result in massive intraoperative bleeding. The technical difficulty of radical excision of these lesions may lead to a relatively high risk of incomplete resection or perioperative complications such as cerebrospinal fluid 
leakage, postoperative bleeding, and cranial nerve palsy.,19 Therefore, the benefits and hazards of aggressive surgery should always be considered for this disease with benign histology but clinically aggressive behavior. In light of the rapid recurrence after resection and high potential morbidity associated with aggressive surgery, adjuvant treatment such as radiation, chemotherapy, or antiangiogenic therapy might have some potential roles for the treatment of IPEH even though there have been no clinical trials. Considering the highly vascular nature of the disease, preoperative embolization is useful for limiting intraoperative bleeding. IPEH should be considered as a potential diagnosis when encountering complex lesions in the base of the skull, such as in the petrous or cavernous sinus, or in the hemispheres that appears to be associated with vascular malformations or structures. The natural history of intracranial IPEH may be more aggressive than previously believed, and long-term follow-up studies on this disease are needed. The results of the present case indicate that optimal resection with multimodal adjuvant treatment can control the disease even over long-term follow-up.

\section{References}

1. Avellino AM, Grant GA, Harris AB, Wallace SK, Shaw $\mathrm{CM}$ : Recurrent intracranial Masson's vegetant intravascular hemangioendothelioma. Case report and review of the literature. J Neurosurg 91:308-312, 1999

2. Baylor JE, Antonelli PJ, Rojiani A, Mancuso AA: Facial palsy from Masson's vegetant intravascular hemangioendothelioma. Ear Nose Throat J 77:408-410, 415-417, 1998

3. Cagli S, Oktar N, Dalbasti T, Işlekel S, Demirtaş E, Ozdamar $\mathrm{N}$ : Intravascular papillary endothelial hyperplasia of the central nervous system - four case reports. Neurol Med Chir (Tokyo) 44:302-310, 2004

4. Chen TJ, Kuo T: Giant intracranial Masson's hemangioma. Report of a fatal case. Arch Pathol Lab Med 108:555-556, 1984

5. Duong DH, Scoones DJ, Bates D, Sengupta RP: Multiple intracerebral intravascular papillary endothelial hyperplasia. Acta Neurochir (Wien) 139:883-886, 1997

6. Hagiwara A, Inoue Y, Shakudo M, Wakasa K, Sato K, Ohata $\mathrm{K}$, et al: Intracranial papillary endothelial hyperplasia: occurrence of a case after surgery and radiosurgery. J Comput Assist Tomogr 23:781-785, 1999

7. Izukawa D, Lach B, Benoit B: Intravascular papillary endothelial hyperplasia in an intracranial cavernous hemangioma. Neurosurgery 21:939-941, 1987

8. Kristof RA, Van Roost D, Wolf HK, Schramm J: Intravascular papillary endothelial hyperplasia of the sellar region. Report of three cases and review of the literature. J Neurosurg 86:558-563, 1997

9. Lesley WS, Kupsky WJ, Guthikonda M: Intravascular papil- lary endothelial hyperplasia arising within a posteroinferior cerebellar artery aneurysm: case report and review of the literature. Neurosurgery 47:961-966, 2000

10. Levere SM, Barsky SH, Meals RA: Intravascular papillary endothelial hyperplasia: a neoplastic "actor" representing an exaggerated attempt at recanalization mediated by basic fibroblast growth factor. J Hand Surg Am 19:559-564, 1994

11. Masson MP: Hémangioendothéliome végétant intravasculaire. Bull Soc Anat (Paris) 93:517-523, 1923

12. Nagib MG, Sung JH, Seljeskog EL: Neurocutaneous Masson's vegetant intravascular hemangioendothelioma. Neurosurgery 11:800-803, 1982

13. Patt S, Kaden B, Stoltenburg-Didinger G: Intravascular papillary endothelial hyperplasia at the fissura orbitalis superior: a case report. Clin Neuropathol 11:128-130, 1992

14. Shih CS, Burgett R, Bonnin J, Boaz J, Ho CY: Intracranial Masson tumor: case report and literature review. J Neurooncol 108:211-217, 2012

15. Sickler GK, Langford LA: Intracranial tumor-forming papillary endothelial hyperplasia-a case report. Clin Neuropathol 9:125-128, 1990

16. Stoffman MR, Kim JH: Masson's vegetant hemangioendothelioma: case report and literature review. J Neurooncol 61:17-22, 2003

17. Tsuji N, Tsubokawa T, Katayama Y, Yamamoto T, Nemoto N: Arteriovenous malformation occluded by Masson's vegetant intravascular haemangioendothelioma and complicated with intracerebral haematoma. Neurol Res 16:148-150, 1994

18. Wen DY, Hardten DR, Wirtschafter JD, Sung JH, Haines SJ: Elevated intracranial pressure from cerebral venous obstruction by Masson's vegetant intravascular hemangioendothelioma. Case report. J Neurosurg 75:787-790, 1991

19. Zhang R, Zhou LF, Mao Y, Wang Y: Papillary endothelial hyperplasia (Masson tumor) of the petrous and jugulare region: case report and literature review. Surg Neurol 64:5560,2005

\section{Author Contributions}

Conception and design: Cho. Acquisition of data: Sim, Lim, Won. Analysis and interpretation of data: Sim, Lim. Drafting the article: Sim. Critically revising the article: all authors. Reviewed submitted version of manuscript: all authors. Approved the final version of the manuscript on behalf of all authors: Cho. Statistical analysis: Sim. Administrative/technical/material support: Cho, Lim. Study supervision: Cho, Lim, Won.

\section{Correspondence}

Kyung Gi Cho, Department of Neurosurgery, Bundang CHA Hospital, CHA University, College of Medicine, 351 Yatap-dong, Bundang-gu, Seongnam, Gyeonggi-do 463-712, Korea. email: sandori50@gmail.com. 